\title{
Test-retest strength reliability of the Electronic Push/Pull Dynamometer (EPPD) in the measurement of the quadriceps and hamstring muscles on a new chair
}

\author{
Mikhled F. Maayah ${ }^{1,2^{*}}$, Mohammad D. Al-Jarrah ${ }^{1}$, Saad S. El Zahrani ${ }^{3}$, Ali H. Alzahrani ${ }^{4}$, \\ Emad T. Ahmed ${ }^{2}$, Amr A. Abdel-Aziem², Gopichandran Lakshmanan ${ }^{5}$, Nabeel A. Almawajdeh ${ }^{5}$, \\ Muhsen B. Alsufiany ${ }^{2}$, Yaser O. M. Abu Asi ${ }^{2}$ \\ ${ }^{1}$ Department of Physiotherapy, Jordan University of Science and Technology, Irbid, Jordan \\ ${ }^{2}$ The Department of Physical Therapy, Faculty of Applied Medical Science, Taif University, Taif, KSA \\ ${ }^{3}$ Department of Family Medicine, Taif University, Taif, KSA \\ ${ }^{4}$ Department of Preparatory Year, Faculty of Applied Medical Science, Taif University, Taif, KSA \\ ${ }^{5}$ The Department of Nursing, Faculty of Applied Medical Science, Taif University, Taif, KSA \\ Email: ${ }^{*}$ mikhledm@just.edu.jo
}

Received 29 October 2011; revised 11 December 2011; accepted 29 January 2012

\begin{abstract}
Background: Test-retest strength reliability of the Electronic Push/Pull Dynamometer (EPPD) in the measurement of the extensor and flexor muscles on a new constructed chair. The objective of the study was to assess reliability of Electronic Push/Pull Dynamometer in the measurement of the knee flexion and extension at $90^{\circ}$ and $60^{\circ}$ on a new constructed chair. The aims of the author: To assess reliability of Electronic Push/Pull Dynamometer in the measurement of the knee flexion and extension at $90^{\circ}$ and $60^{\circ}$ on a new constructed chair. Design: A test-retest reliability study. Subjects: One hundred healthy students male and female (mean age, 21 y). Methods: Maximum isometric strength of the quadriceps and hamstring muscle groups was measured using the EPPD were recorded at $60^{\circ}$ and $90^{\circ}$ for 3 trials on 2 occasions. Reliability was assessed with the Intraclass correlation coefficient (ICC), mean and standard deviation (SD) of measurements, and smallest real differences were calculated for the maximum and for the mean and work of the 3 repetitions. Results: Mean strength ranged from $50.44 \mathrm{~kg}$ for knee flexion to $55.76 \mathrm{~kg}$ for knee extension $50.44 \mathrm{~kg}$ to $61.98 \mathrm{~kg}$ at $90^{\circ}$ hip flexion. Test-retest reliability Intraclass correlation coefficients (ICCs) ranged from 0.85 to 0.99 . ICCs for test-retest reliability ranged from 0.780 to 0.998 . Conclusions: The results of the reliability study indicate that the EPPD in reliable dynamometer to use in determining lower limb muscle force production. It can be used to measure disease progression and to evalu-
\end{abstract}

"Corresponding author. ate changes in knee extension and flexion strength at the individual patient level.

Keywords: Muscle Strength; Reliability; Test-Retest; Hand-Held Dynamometer; Electronic Pull/Push Dynamometer

\section{INTRODUCTION}

Muscle strength in different positions of the knee joint is an important factor in evaluating the joint in rehabilitation settings. Most of the published articles used hand-held dynamometer and manual muscle testing to evaluate the muscle strength of the knee joint; quadriceps and hamstrings in particular. The Electronic Push/Pull Dynamometer (EPPD) is a hand-held dynamometer, which has gained popularity in measuring muscle strength in clinical practice because of its simplicity and objectivity [1-5]. Clinically, EPPD is a widely used tool for measuring muscle and has been found to correlate with isokinetic strength scores [6]. The test-retest reliability of the HandHeld dynamometer in 41 community-dwelling patients (23 females and 18 males), with a mean age of 76 (1.2) years was evaluated by Wang et al. [7]. He found the Hand-Held dynamometer reliable for the lower extremity limbs in community dwelling elderly patients with a history of falling. The test-retest interclass correlation coefficient ranged from 0.95 to 0.99 for 1 trial and from 0.97 to 1.0 for the mean of 2 trials. Reed, Den Hartog et al. [8] investigated the relationship between MFG isometric strength scores and isokinetic strength scores in 82 healthy elderly individuals 60 years of age and older. They found a strong association $(r=0.77-0.85)$ between the two measurement approaches, but Hand-Held dy- 
namometer isometric scores were found to be variable. Reed attributed the measurement variability to the lack of stability of the Hand-Held dynamometer and recommended the use of fixed instrumentation for clinical studies for greater accuracy.

The Department of Biomedical Engineering of Virginia Commonwealth University, Medical College of Virginia Campus (Figure 1), designed a portable steel frame for use with the Mecmesin Force Gauge (MFG) [9]. The frame was constructed with a moveable arm, which can be adjusted for limb length differences and a rotating mechanism to hold the frame in place during an isometric contraction. The frame can accept up to $330 \mathrm{~K}$ of force with the brakes locked on a carpeted surface. The disadvantage of this technique is that the frame is heavy and not portable for use it in different locations. In 2002 the present investigator designed a portable frame to which the MFG could be attached in the School of Physiotherapy at Curtin University of Technology, Perth (Figure 2). The frame was constructed with a moveable, sliding, adjustable arm support, which can be adjusted for limb length. The adjustable arm can be removed to change the position of the MFG from one side to the other. The examiner can easily reposition the frame arm and MFG to allow for different muscle testing position. In 2011 the same investigator designed a portable base fixed on the exercise knee extension machine (pin loaded) to which the EPPD

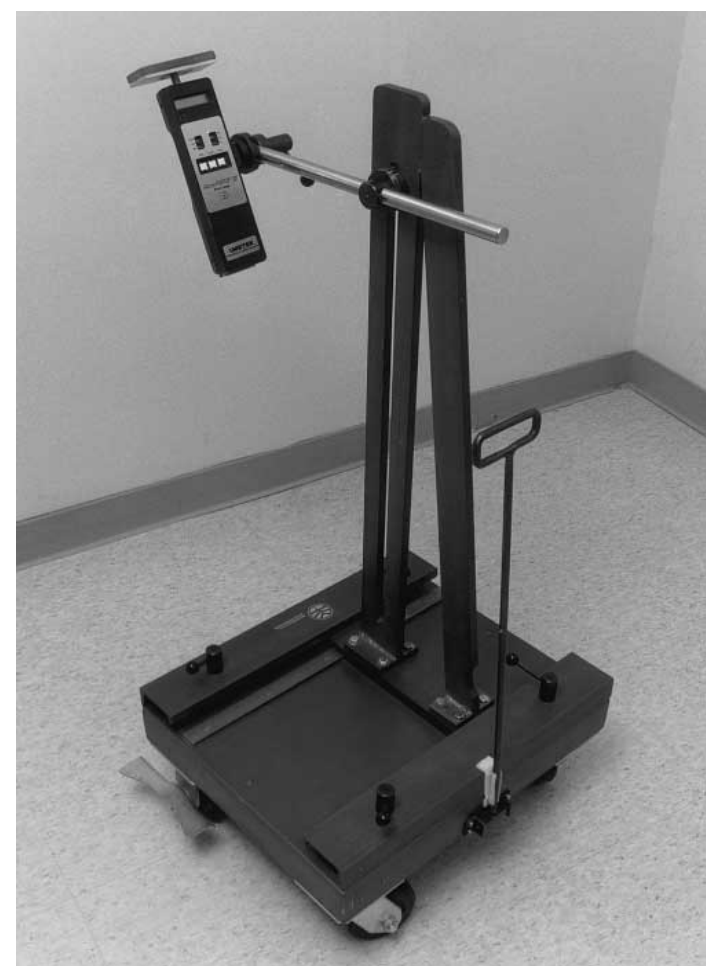

Figure 1. AccuForce II Digital Force Gage attached to a portable steel frame with movable arm and rotating disc and braking mechanism. could be attached in the physiotherapy department at Jordan University of Science and technology, Irbid (Figure 3). The EPPD was constructed with a movable base, sliding, adjustable arm base support, which can be adjusted for limb length. The base of the EPPD can be removed to change the position of the device from one

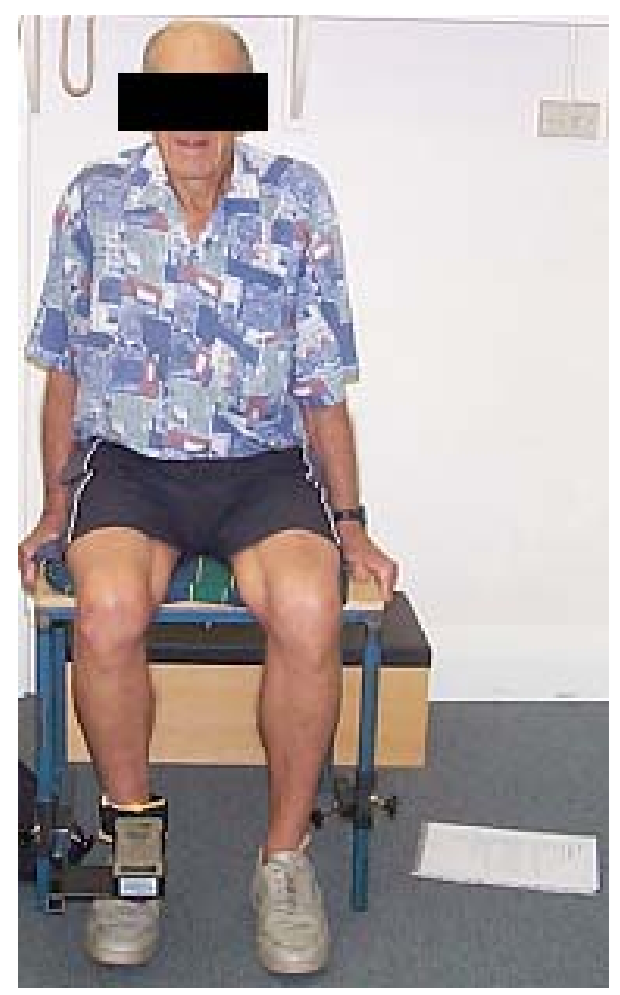

Figure 2. Electronic Pull/push Dynamometer attached to a portable constructed chair to measure muscle strength.

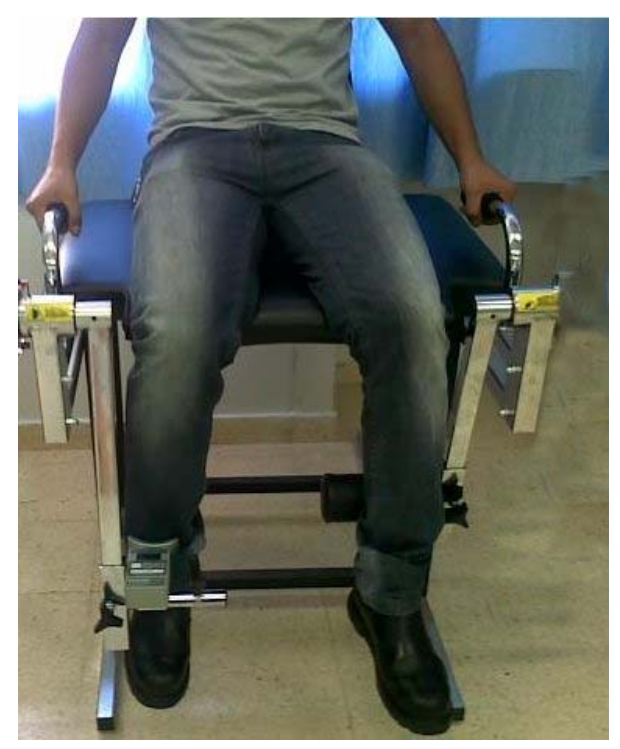

Figure 3. Electronic Pull/push Dynamometer attached to on a new constructed chair. 
side to the other. The advantage of the new designed is that the patient can seat it with backrest, whereas, in Figure 2, the chair was not have back support. The main objective of this study was to examine the reliability of the EPPD on a new designed chair.

\section{MATERIAL AND METHODS}

\subsection{Materials}

Base line of Electronic Push/Pull Digital Dynamometer with $2501 \mathrm{~b} / 120 \mathrm{Kg}$ capacity, which can measure muscle strength in kilograms (kg) or in pounds (lb) .This device, has two ends one side is push and another side is pull. It has only 5 buttons that is on/off, zero, max, max clear, 1 $\mathrm{b} / \mathrm{kg}$. On/off button for switching on and off the equipment, zero buttons to ensure that you are starting from zero measurement, max button to memorize the last maximum reading, max clear button to clear the last maximum reading and $\mathrm{lb} / \mathrm{kg}$ button is to shift either to pounds or kilograms. Machine is battery operated and requires two AAA batteries to work. Two inch wide digital screen shows the strength measurement, battery life and $\mathrm{lb}$ or $\mathrm{kg}$ according to choice (Figure 4). Inch tape, weighing scale, rolled towel padding and a couch are the other required materials. Handles and accessories are interchangeable with the Baseline hydraulic pushpull dynamometer: Large curved pad; Straight pad; $1 \mathrm{~cm}^{2}$ circ padded; Medium hook and Oval snap hook.

\subsection{Individuals}

One hundred healthy adult male and female physiotherapy students were recruited from the department of physiotherapy in Allied Medical Sciences at Jordan University of Science and Technology. Individual subjects attending reliability session were in between 18 - 35 year of age were included in the study. Participants were excluded with at least one of the following factors: Presence of other active rheumatic disease (e.g. rheumatoid, septic arthritis, gouty arthritis); Medical history of neuromuscular or neurological disease (e.g. stroke, Parkinson's disease); medically unstable; lower limb joint replacement and Musculoskeletal disease. A sample size of 100 participants was selected. Written informed consent was obtained from club officials and individual participants. Ethical approval was obtained prior to the commencement

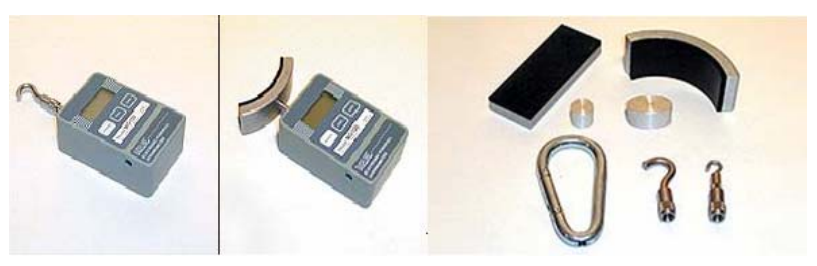

Figure 4. Baseline hand held dynamometer. of the study from the Human Research Ethics Committee of Jordan University of science and Technology.

\subsection{Procedures}

Each individual was tested on 2 separate occasions at the same time of the day (e.g. 10 am - 2 pm), 1 week apart. The same examiner performed all measurements. The total time for a test session was approximately 5 minutes.

\section{Isometric Muscle Strength Measurements}

Maximal isometric strength of the knee extensors (quadriceps muscle) and flexors (hamstring muscle) was measured using the EPPD. This digital strain gauge dynamometer displays the force measurement to the nearest $0.1 \mathrm{~kg}$, up to a maximum of $199.9 \mathrm{~kg}$. Measurements were used at $60^{\circ}$ and $90^{\circ}$ of knee flexion. Prior to each episode of measurement, the instrument was calibrated according to the manufacturer's instructions and specifications.

The individuals were seated in a comfortable position with the backrest angled at $100^{\circ}$ to the seat without shoes or orthotic device. Before each measurement the full range of motion (ROM) was set and the dynamometer was applied. The dynamometer position was standardized with specific landmarks. The shin pad was placed 2 $\mathrm{cm}$ between the above the medial and lateral malleoli. The instrument shaft remained horizontal to the anterior aspect of the mid shaft of tibia and horizontal to the posterior aspect over the musculotendinous junction of calf muscles. Subjects were then asked to maintain the limb in the stipulated position and to hold that position while pushing or pulling against the dynamometer. Subjects were asked to push or pull against the gauge pad as hard as possible when given the appropriate command. All measurements were performed with the limb segment in a position that was with gravity eliminated. Rests between trials were approximately 30 seconds. Each contraction was held for six seconds, and the subject was encouraged to breathe normally to avoid any increase in heart rate or blood pressure. Before measuring each muscle group, the examiner explained to the subject the contraction to be performed. Re-tests of the specific muscle groups were performed 1 week after the initial measurement.

Two variables were extracted for each of the knee direction (flexion, extension) and isometric strength was measured in kilograms. The procedure was performed three times. The highest output was selected and recorded throughout the range of motion of each repetition Wang et al. [7]. For each muscle action the limb was placed in the appropriate angle over the edge of the newly constructed chair (Figure 1). The testing positions for knee flexors and extensors were sitting with knee and hip flexed to 90 degree. 


\subsection{Ethical Consideration Aspects}

The study was approved by the Human Research Ethics Committee of Jordan University of science and Technology. Confidentially was maintained by restricting access to data, which stored on a password protected computer hard disk. All subject information was coded with each participant being identified by number only and only one supervisor was hold the master list. All materials were stored in a secure archive at Jordan University of science and technology for seven years.

\subsection{Data Analysis}

All data analysis was performed with the SPSS, version 17, for Windows statistical program. Descriptive analysis was conducted on the demographic variables. Means were calculated for each muscle group for both knee flexion and extension at $90^{\circ}$ and $60^{\circ}$ were used in the data analysis separately. The infraclass correlation coefficients (ICCs) and the $90 \%$ confidence intervals (CIs) were determined for the individual scores. To determine the reliability of the knee flexion and extension at $90^{\circ}$ and $60^{\circ}$, ICCs were estimated along with $90 \%$ confidence intervals.

\section{RESULTS}

One hundred healthy physiotherapy students were invited to participate in the reliability study. All subjects who were approached agreed to participate and all of them completed the testing session. The age of participants ranged from 18 to 35 years (mean 21.31, SD. 1.9).

Table 1 presents the test and re-test peak isometric force production scores for all subjects. The scores have been presented for knee flexor and extensors muscle at $90^{\circ}$ and $60^{\circ}$ degree. Electronic Pull/Push Dynamometer scores differed little between the knee extensors and flexors strength at $90^{\circ}$ and $60^{\circ}$ during the test or re-test sessions in female subjects. Knee extensors (quadriceps) force production was the highest in both female and male, whereas knee flexors performance (hamstring) was the lowest average force scores. Subjects showed higher re-test scores strength throughout the extensors and flexors muscles group. The T-Test results showed no significant in test and re-test separately in knee flexion and extension at $90^{\circ}$ and $60^{\circ}$ in both male and female. The re-test was at 1 week later of test.

Table 2 shows test and re-test peak isometric force production scores for knee extension and flexion at $90^{\circ}$ and $60^{\circ}$. The T-Test results showed a highly significant in comparison between male and female in test and re-test.

Table 3 shows the ICCs for individual mean knee flexors and extensors scores. The ICCs ranged from 0.85 to 0.99 in both muscle groups. The ICCs in knee extension and flexion at $90^{\circ}$ revealed greater scores than knee extension and flexion at $60^{\circ}$. All the participants enrolled in the study completed the reliability study measurements twice, one week apart.

Table 1. Test-retest reliability of isometric strength testing using Electronic Pull/Push Dynamometer.

\begin{tabular}{|c|c|c|c|c|}
\hline Gender & Muscle Group Action & Test & Re-Test & P Value \\
\hline \multirow{4}{*}{ Female } & Knee Extension at $90^{\circ}$ & $46.89(23.26)$ & $46.72(23.29)$ & $0.97 \mathrm{NS}$ \\
\hline & Knee Extension at $60^{\circ}$ & $39.23(19.31)$ & $38.87(19.14)$ & $0.77 \mathrm{NS}$ \\
\hline & Knee Flexion at $90^{\circ}$ & $42.48(17.50)$ & $42.69(17.53)$ & $0.90 \mathrm{NS}$ \\
\hline & Knee Flexion at $60^{\circ}$ & $42.53(17.48)$ & $38.07(19.14)$ & $0.25 \mathrm{NS}$ \\
\hline \multirow{4}{*}{ Male } & Knee Extension at $90^{\circ}$ & $74.70(22.40)$ & $73.48(21.47)$ & $0.77 \mathrm{NS}$ \\
\hline & Knee Extension at $60^{\circ}$ & $64.72(17.78)$ & $60.79(17.81)$ & $0.26 \mathrm{NS}$ \\
\hline & Knee Flexion at $90^{\circ}$ & $66.39(18.43)$ & $66.64(18.47)$ & $0.90 \mathrm{NS}$ \\
\hline & Knee Flexion at $60^{\circ}$ & $66.48(18.22)$ & $60.79(17.81)$ & $0.11 \mathrm{NS}$ \\
\hline
\end{tabular}

Data are represented as mean (SD), significance were considered at $\mathrm{P} \leq 0.05$ using $t$-Test $(\mathrm{n}=46$ female $\& 54$ male).

Table 2. Test-Retest Peak Isometric Force Production Scores $(\mathrm{kg})$ for knee extensors and flexors $(\mathrm{n}=100)$.

\begin{tabular}{|c|c|c|c|c|c|c|}
\hline \multicolumn{4}{|c|}{ TEST } & \multicolumn{3}{|c|}{ RETEST } \\
\hline & Male & Female & P Value & Male & Female & P Value \\
\hline Knee Extension at $90^{\circ}$ & $74.70(22.40)$ & $46.89(23.26)$ & 0.001 & $73.48(21.47)$ & $46.72(23.29)$ & 0.001 \\
\hline Knee Extension at $60^{\circ}$ & $64.72(17.78)$ & $39.23(19.31)$ & 0.001 & $60.79(17.81)$ & $38.07(19.14)$ & 0.001 \\
\hline Knee Flexion at $90^{\circ}$ & $66.39(18.38)$ & $42.48(17.50)$ & 0.001 & $66.64(18.47)$ & $42.69(17.53)$ & 0.001 \\
\hline Knee Flexion at $60^{\circ}$ & $66.48(18.22)$ & $39.23(19.31)$ & 0.001 & $60.79(17.81)$ & $38.07(19.14)$ & 0.001 \\
\hline
\end{tabular}

Data are represented as mean (SD), significance were considered at $\mathrm{P} \leq 0.05$ using $t$-Test $(\mathrm{n}=46$ female \& 54 male). 
Table 3. Reliability of knee flexors and extensors scores at $90^{\circ}$ and $60^{\circ}(\mathrm{n}=100)$.

\begin{tabular}{ccc}
\hline Knee Force & ICCs & $\mathbf{9 0 \% ~ C I ~}$ \\
\hline Knee Extension at $90^{\circ}$ & 0.99 & $0.987-0.994$ \\
Knee Extension at $60^{\circ}$ & 0.93 & $0.893-0.950$ \\
Knee Flexion at $90^{\circ}$ & 0.99 & $0.995-0.998$ \\
Knee Flexion at $60^{\circ}$ & 0.85 & $0.847-0.894$ \\
\hline
\end{tabular}

\section{DISCUSSION}

This is the first study to investigate the reliability of Electronic Pull/Push Dynamometry for muscle strength testing in healthy students in a new chair. The main finding of this study was that the test-retest reliability coefficients exceeded 0.78 for all variables, suggesting that the EPPD has appropriate reliability for such studies. All ICCs calculated for test-retest reliability in this study were "excellent".

The advantages of the EPPD are its portability and the ease of its use as no attachments needed to measure upper and lower extremity muscle groups.

However, the disadvantage of the Hand-Held is that the reliability of the measurements depends on the strength of the examiner and their ability to maintain the testing position while holding against the resistance of a subject.

These findings are in agreement with previous studies on western populations demonstrating excellent test-retest reliability for these outcome measures in adult healthy physiotherapy students using the EPPD [9-14]. These values are much higher than those reported by Cheryl et al. [9] of 25 (17 women and 8 men) community dwelling older adults aged between 70 to 87 years of age with confirmed OA of the knee, the mean knee extension values measured at $90^{\circ}$ were $(20.3 \pm 11.7)$ and $(20.4 \pm 11.0)$ $\mathrm{kg}$ for the right and left knees respectively. Whereas, the mean of this study in knee extension values measured at $90^{\circ}$ was 61.98 (26.57) and at $60^{\circ}$ was 53.12 (22.47).

Test-retest reliability of knee flexor strength using EPPD tended to be higher in this study than that observed in nonathletic populations, where ICCs as high as 0.965 have been observed [15]. The mean strength values measured during our study were considerably higher than those found in other studies measuring strength of these muscle groups using EPPD. Our highest average knee flexion measurement of $55.53 \mathrm{~kg}$ and maximum recording of $55.56 \mathrm{~kg}$ is significantly greater than the maximum value of $21 \mathrm{~kg}$ [16].

Greater strength of muscle groups has already been shown to adversely affect the reliability of HHD testing $[17,18]$. In the current study, reliability measures were highest for measures of knee flexor strength and knee extensor at $90^{\circ}$ hip flexion, representing the strongest muscle groups. One of the strengths of this study is that it involved testing positions that have been validated for the assessment of students. These tests are performed in the functional range of the healthy physiotherapy students, which makes them more relevant to clinical practice.

Some limitations of the current study should be noted. The study samples represented a convenience sample and were not randomly or systematically selected. In addition, the investigator was not blind to the results of earlier tests, introducing the possibility of bias. Furthermore, all measurements were conducted during a single session in two occasions by the same investigator. Electronic Push/ Pull Dynamometer isometric flexor and extensor strength can be performed with good to excellent test-retest reliability when measured by the same examiner.

\section{CONCLUSION}

The results of the reliability study indicate that the EPPD in reliable dynamometer to use in determining lower limb muscle force production. It can be reliable dynamometer to use in measurement of muscle strength in healthy subject age 18 - 35 year. It can be used to measure disease progression and to evaluate changes in knee extension and flexion strength at the individual patient level. Testing of knee flexor and extensor strength using Electronic Pull/Push Dynamometry in healthy adult students can be done with excellent reliability.

\section{ACKNOWLEDGEMENTS}

The authors thanks the physiotherapy students who participated in the study, the help and support of the physiotherapy staff for the valuable input in this study and Prof. Dr. Osama Mohamed S. Abo-Salem for statistical help.

\section{REFERENCES}

[1] Bohannon, R.W. (1986) Manual muscle test scores and dynamometer test scores of knee extension strength. Archives of Physical Medicine and Rehabilitation, 67, 390392.

[2] Wadsworth, C.T., Krishnan, R., Sear, M., et al. (1987) Intrarater reliability of manual muscle testing and handheld dynamometric muscle testing. Physical Therapy Reviews, 67, 1342-1347.

[3] Noreau, L. and Vachon, J. (1998) Comparison of three methods to assess muscular strength in individuals with spinal cord injury. Spinal Cord, 36, 716-723. doi:10.1038/sj.sc.3100646

[4] Schwatz, S., Cohan, M.E., Herbison, G.J., et al. (1992) Relationship between two measures of upper extremity strength: Manual muscle test compared to hand-held myometry. Archives of Physical Medicine and Rehabilitation, 73, 1063-1068.

[5] Arnold, C., Warkentin, K.D., Chilibeck, P.D. and Magnus, 
C.R. (2009) The reliability and validity of handheld dynamometry for the measurement of lower-extremity muscle strength in older adults. The Journal of Strength \& Conditioning Research, 24, 815-824.

[6] Tan, K.H., Balci, N., Sepsi, V. and Gender, F.A. (1995) Isokinetic and isometric strength in osteoarthritis of the knee: A comparative study with healthy women. American Journal of Physical Medicine and Rehabilitation, 74, 364-369.

[7] Al-Arfaj, A. and Al-Boukai, A. (2002) Prevalence of radiographic osteoarthritis of the hands in Saudi Arabia. Rheumatology Internal, 22, 208-212. doi:10.1007/s00296-002-0228-5

[8] Reed, R.L., Den Hartog, R., Yochum, K., et al. (1993) A comparison of hand-held isometric strength measurement with isokinetic muscle strength measurement in the elderly. Journal of America and Geriatric Society, 41, 5356.

[9] Cheryl, D., Ford-Smith, P.T., et al. (2001) Reliability of stationary dynamometer muscle strength testing in community dwelling older adults. Archives Physical Medicine \& Rehabilitation, 82, 1128-1132. doi:10.1053/apmr.2001.24291

[10] Brosseau, L., Tousignant, M., Budd, J., Chartier, N., Duciaume, L., Plamondon, S., O'Sullivan, J.P., O'Donoghue, S. and Balmar, S. (1997) Intratester and intertester reliability and criterion validity of the parallelogram and universal goniometer for active knee flexion in healthy subjects. Physiotherapy Research International, 2, 150-166. doi:10.1002/pri.97

[11] Brosseau, L., Casimiro, L., et al. (2001) Therapeutic ultrasound for treating petellofemoral pain syndrome. Cochrance Data base Systmatic Review, 4, Article ID: CD003375.
[12] Brazier, J.E., Harper, R., Munro, J., Walters, S.J. and Snaith, M.L. (1999) Generic and condition-specific outcome measures for people with osteoarthritis of the knee. Rheumatology (Oxford), 38, 870-877. doi:10.1093/rheumatology/38.9.870

[13] Faucher, M., Lefevre-Colau, M.M., Rannou, F., Fermanian, J. and Revel, M. (2003) Assessment of the test-retest reliability and construct validity of a modified Lequesne index in knee osteoarthritis. Joint Bone Spine, 70, 521-525. doi:10.1016/S1297-319X(03)00070-8

[14] Salaffi, F., Carotti, M., Stancati, A. and Grassi, W. (2003) Radiographic assessment of osteoarthritis: Analysis of disease progression. Aging Clinical and Experimental Research, 15, 391-404.

[15] Bohannon, R. (1997) Reference values for extremity muscle strength obtained by hand-held dynamometry from adults aged 20 - 79 years. Archives of Physical Medicine and Rehabilitation, 78, 26-32. doi:10.1016/S0003-9993(97)90005-8

[16] Andrews, A., Thomas, M.W. and Bohannon, R.W. (1996) Normative values for isometric muscle force measurements obtained with hand-held dynamometers. Physical Therapy, 76, 248-259.

[17] Brinkmann, J. (1994) Comparison of hand-held and fixed dynamometer in measuring strength of patients with neuromuscular disease. The Journal of Orthopaedic \& Sports Physical Therapy, 19, 100-104.

[18] Agre, J., Magness, J.L., Hull, S.Z., et al. (1987) Strength testing with a portable dynamometer: Reliability for upper and lower extremities. Archives of Physical Medicine and Rehabilitation, 68, 454-458. 